\title{
On the relationship between automation and occupants in smart buildings
}

\author{
Ilche Georgievski \\ Distributed Systems Group \\ Johann Benroulli Institute \\ for Mathematics and Computer Science \\ University of Groningen \\ Email: i.georgievski@rug.nl
}

\author{
Thijs Bouman \\ Environmental Psychology Group \\ Faculty of Behavioural and Social Sciences \\ University of Groningen \\ Email: t.bouman@rug.nl
}

\section{EXTENDED ABSTRACT}

Cities are significant contributors to global emission of $\mathrm{CO}_{2}$. Among city entities, buildings are the largest energy consuming sector and are therefore an important source of $\mathrm{CO}_{2}$ emissions. A key issue in buildings is that their current energy systems fail to use energy in a smart and sustainable way. That is, energy-use policies (e.g., with regard to lights in public spaces) and individuals energy behaviours (e.g., switching lights on/off in personal spaces) are often inefficient. In the context of the BeijIng Groningen Smart energy cities (BIGS) project, ${ }^{1}$ we aim to optimise building energy systems through the automation of certain aspects of the building energy system (phase 1 of project, 2015-2016), while using this platform to engage end-users by interfacing them with the automated solution (phase 2 of the project, 2017-2018).

The goal of the BIGS project is to create efficient and sustainable future smart energy systems through the design and implementation of an intelligent ICT platform that includes sensors, actuators and techniques for context reasoning and automation. Importantly, instead of just silently taking over energy decisions through automation, our approach focuses on how to get users involved as well. Therefore, we aim to understand people's energy behaviours and to create policies that improve people's behaviours and sustainability by using appropriate ICT solutions. This necessitates a holistic approach that takes into account knowledge on information technology and reasoning, human decision making (motivations, values, incentives) and human behaviour.

In the first phase of BIGS, we focus on testing an ICT platform with automation in a real environment, and understanding people's awareness of and attitudes toward the use of automation. For this purpose, we use the restaurant of the Bernoulliborg building at the Zernike campus of the University of Groningen, The Netherlands, to model and control the lamps automatically. The restaurant covers an area of 251.50 $\mathrm{m}^{2}$ with a capacity of 200 sitting places. The restaurant has glass walls from three sides, enabling a significant amount of natural light to come through when the weather conditions allow for it. The restaurant area is used for lunch and, outside

\footnotetext{
${ }^{1}$ http://jstp-bigs.com/
}

lunch hours, the area is used by staff, students or other visitors for working, meeting, or other purposes. We use movement sensors, a natural light sensor and the physical properties of the restaurant to model and interpret the context. We then employ an artificial intelligence planning technique to automatically coordinate the use of lamps given the current context and the goal to keep user comfortable under constraints of energy efficiency. The control of lamps is enabled by actuators attached to each lamp. We deployed and run the solution for several months. When compared to the previous way of controlling the lamps in the restaurant, which involves turning on and off lamps manually at fixed points in the morning and evening of each day, our solution achieves savings in the order of $80 \%$ of energy [1].

Within this context, we also performed preliminary user studies. These studies indicated that most restaurant occupants self-reported that they are aware of sustainability issues and engage in environmentally friendly behaviours. In addition, most of those restaurant occupants indicated that they would accept an intelligent system as the one we proposed and that they would be satisfied with such a solution. However, with regard to the actual solution, only about one third of the occupants indicated being aware of the solution, and their involvement in and influence on the solution was low. We believe this lack of awareness and involvement is a commonly missed opportunity, considering that behavioural research indicated that making people aware of their involvement in sustainable behaviours (e.g., their acceptance and contribution to a solution like this) predict future pro-environmental behaviours and attitudes, particularly for those who indicate to value the environment [2], [3].

In the second phase of BIGS, we will address this issue and focus on identifying energy-related preferences, needs and behaviours of individuals, and look at how interfacing ICT solutions with end-users could motivate and empower people in their everyday use of energy. Moreover, we will work on centering automated techniques around individuals by taking into account their needs, preferences, and motivations, which is essential for the creation of successful and smart energy systems that truly empower end-users. This will enable us to understand the needs of people during the design and develop- 
ment process, shape the system design to their requirements, and engage them in future energy efficient behaviours.

\section{REFERENCES}

[1] I. Georgievski, "Coordinating services embedded everywhere via hierarchical planning," Ph.D. dissertation, University of Groningen, October 2015.

[2] L. Steg, G. Perlaviciute, and E. van der Werff, "Understanding the human dimensions of a sustainable energy transition," Frontiers in Psychology, vol. 6 , no. 805, 2015.

[3] E. van der Werff, L. Steg, and K. Keizer, "I am what i am, by looking past the present: The influence of biospheric values and past behavior on environmental self-identity," Environment and Behavior, vol. 46, no. 5, pp. 626-657, 2014. 